\title{
IL-17 regulates the expression of major histocompatibility complex II and VEGF in DLBCL mice on tumor growth
}

\author{
Xin $\mathrm{Xu}^{\mathrm{a},{ }^{*}}$, Ting Wei ${ }^{\mathrm{b}}$, Weijie Zhong ${ }^{\mathrm{a}}$, Zhigang Zhu ${ }^{\mathrm{a}}$, Feng Liu ${ }^{\mathrm{a}}$, Qingshan $\mathrm{Li}^{\mathrm{b}}$ \\ a Department of Geriatrics, Hematology \& Oncology ward, Guangzhou First People's Hospital, School of Medicine, \\ South China University of Technology, Guangzhou, Guangdong, P.R.510180, China. \\ ${ }^{b}$ Department of Hematology, Guangzhou First People's Hospital, School of Medicine, South China University of Technol- \\ ogy, Guangzhou, Guangdong, P.R.510180, China.
}

\begin{abstract}
Objective: To investigate the effect of interleukin-17 (IL-17) on vascular endothelial growth factor (VEGF) and major histocompatibility complex II (MHC II) expression in diffuse large B-cell lymphoma (DLBCL) and the relationship thereof with tumor development.

Methods: CD4 + CD62L + naïve cells from spleen of BALB/c mice were purified by the immunomagnetic beads method. T-cells were cultured in vitro with anti-CD3, anti-CD28, transforming growth factor- $\beta$ (TGF- $\beta$ ), and interleukin-6 (IL-6). The human germinal center B-cell-like diffuse large B-cell lymphoma (GCB-DLBCL) cell line SUDHL-4 was cultured and inoculated into severe combined immune deficiency (SCID) mice to establish a DLBCL mice model. Tumor-bearing mice were inoculated with Th17 cells. DLBCL was detected by ELISA. The expression of IL-17, VEGF, and MHC II were detected by immunohistochemistry (IH).

Results: After Th17 cells were inoculated, the IL-17 expression level was higher than that of the control group, the VEGF expression level as lower than that of the control group, and the MHC II expression level was higher than that of the control group. There was a significant negative correlation between IL-17 and VEGF, and there was a significant positive correlation between IL-17 and MHC II.

Conclusion: The VEGF and MHC II expression levels can be used as an index to judge the DLBCL disease status. IL-17 can produce negative regulation of VEGF and positive regulation of MHC II, thereby affecting the DLBCL disease process. Detection of IL-17, VEGF, and MHC II expression levels have value in judging the DLBCL disease status and progression.
\end{abstract}

Keywords: Diffuse large B cell lymphoma, Th17, interleukin 17, vascular endothelial growth factor, major histocompatibility complex II

\section{Introduction}

Th17 cells are a new type of helper CD4+ T-cell sub-type that were discovered in recent years. IL-17 is a characteristic cytokine secreted by Th17 cells [1]. IL-17 uses the IL-17 receptor IL-17R [2] in the signal transduction process. Because IL-17R has a single reverse transmembrane domain and cytoplasmic tail region, there are multiple

\footnotetext{
* Corresponding author: Xin Xu

Mailing address: Department of Geriatrics, Hematology \& Oncology ward, Guangzhou First People's Hospital, School of Medicine, South China University of Technology, Guangzhou, Guangdong, P.R.510180, China.

E-mail: eyxuxin@scut.edu.cn

Received: 25 May 2020 / Accepted: 10 June 2020
}

regulatory regions; thus, IL-17 mediates multiple signal pathways.

VEGF is a highly specific factor that can promote endothelial cell division and induce angiogenesis. Its expression is related to angiogenesis, clinicopathological characteristics, and poor prognosis of many tumors, such as those associated with breast and lung cancer. In recent years, it has been reported that the VEGF serum level is an independent prognostic factor for aggressive non-Hodgkin's lymphoma (NHL). The total and disease-free survival (DFS) of patients with high VEGF expression is significantly shorter than that of patients with low VEGF expression [3]. Diffuse large B-cell lymphoma (DLBCL) is the most common invasive NHL. How is VEGF expressed in DLBCL? Can it be regulated by cytokines, such as IL-17, and therefore have different effects on tumor development? At present, there is a lack of in-depth research.

The major histocompatibility complex (MHC) molecule 
is called the human leukocyte antigen (HLA) complex, and it is divided into three sub-types-I, II, and IIIamong which MHC II molecules are mainly expressed on antigen-presenting cells, such as B-lymphocytes, and play a role in regulating cell immunity. In recent years, MHC II molecules have reportedly been related to the pathogenesis of DLBCL. The expression of MHC II in DLBCL and whether MHC II can be regulated by IL-17 and play an important role in the occurrence and development of tumor still lack an in-depth study.

Therefore, this study intends to establish a DLBCL mouse model to compare the expression levels of IL-17, VEGF, and MHC II molecules between the inoculation and control groups and to analyze the correlation between the IL17 level and the expression of VEGF and MHC II and the relationship thereof with tumor development in order to provide an experimental basis for the adoptive immunotherapy of DLBCL by Th17 cells.

\section{Materials and methods}

\section{Th17 cell culture in vitro}

The CD4+ CD62L+ naïve T-cells from the spleen of $\mathrm{BALB} / \mathrm{c}$ mice were isolated and purified by a mini Macs immunomagnetic separation system; the specific operation was carried out in strict accordance with mini Macs instructions. The purity of the CD4+ CD62L + naïve T-cells from the spleen of mice was identified by flow cytometry; when the purity reaches $85-95 \%$, it can be used for in vitro culture. Anti-CD3, anti-CD28, TGF- $\beta$, and IL-6 were added to culture Th17 cells in vitro for three days in a 5\% $\mathrm{CO}_{2}$ incubator at $37^{\circ} \mathrm{C}$, and the growth of the Th17 cells was observed under an inverted microscope.

\section{Establishment of DLBCL mouse model}

Ten SPF grade SCID, female, 5-week-old mice weighing 16-20 g were selected for subculture of the human GCB-like DLBCL cell line SUDHL-4. SUDHL-4 cells were selected in a logarithmic growth stage, and $0.1 \mathrm{ml}$ of 107-containing cells were subcutaneously inoculated in one rib of each mouse. A cell suspension was used to observe the general condition, tumorigenesis, and tumor growth of the mice every day. The body weight and tumor length and height were measured every day, and the tumor volume was calculated (calculation method: $\pi / 6 \times$ length $\times$ width $\times$ height). When the tumor body reached 1200 $\mathrm{mm}^{3}$, it was regarded as the humanity end-point, and the time of tumor occurrence and survival time of each mouse were recorded, in order to obtain the median tumor onset time $\mathrm{T} 1$ and the median survival period $\mathrm{T} 2$ of each mouse. An additional 30 mice were selected to establish a DLBCL mice model according to the above method. Twenty were inoculated with Th17 cells at the same time that they were inoculated with tumor cells; each mouse was injected with Th17 cells $(1.6-2.0) \times 10^{6}$ cells (resuspended with $0.2 \mathrm{ml}$ normal saline) for adoptive immunotherapy experiment of Th17 cells, which was set as group A. The other 10 mice were injected with $0.2 \mathrm{ml}$ normal saline as the control group, which was set as group B.

\section{Detection of IL-17 and VEGF expression in the tumor tissue of DLBCL mice by ELISA}

Half of the mice in groups A and B were killed at T1 and set as groups $\mathrm{A} 1$ and $\mathrm{B} 1$ (10 mice in group $\mathrm{A} 1$ and five mice in group $\mathrm{B} 1$ ); the other half were killed at $\mathrm{T} 2$ and set as groups A2 and B2 (10 mice in group A2 and five mice in group B2). After the mice were killed, the tumor tissue and cell suspension were created by peeling off the tumor and centrifuging it in a centrifuge tube with an equal amount of mouse lymphocyte separation solution added in advance. The tumor mononuclear cell suspension of DLBCL mice was created by adding PBS centrifugation, and the cell supernatant was taken. IL-17 and VEGF expression was detected by a double antibody sandwich enzyme-linked immunosorbent assay (ELISA); the ELISA kits for IL-17 and VEGF were purchased from Shanghai Tongwei company. The minimum detection limits for IL17 and VEGF were $0.1 \mathrm{pg} / \mathrm{ml}$ and $1.0 \mathrm{pg} / \mathrm{ml}$, respectively. Anti-IL-17 and VEGF McAbs were used, respectively, to coat the enzyme plates. The IL-17 and VEGF standards and samples were used to combine with McAbs. Biotinized anti-IL-17 and -VEGF antibodies were added to form the immune complex connection. Finally, the full-automatic enzyme labeling instrument was used for analysis; the operation process was strictly in accordance with the instructions for the ELISA Kit.

\section{Detection of MHC II expression by immunohistochem- istry and determination of results}

The stripped tumor tissue was fixed, paraffin embedded, sectioned, and stained by a routine $\mathrm{HE}$ and immunohistochemistry envision two-step method. MHC II/anti-cd74 is a concentrated anti-human antibody (dilution ratio of 1:500). The positive section in the known pre-experiment was used as the positive control, and PBS was used as the negative control. The result determination was as follows. MHC II was located in the cytoplasm and membrane and judged according to the staining intensity: negative, 0 points; diffuse or scattered dot-like or uniform sediment in the cytoplasm is light brown, 1 point; brown, 2 points; intensive brown, 3 points. It was also judged according to the percentage of positive cells: 0 was negative, 0.5 points for the number of positive cells $<5 \%, 1$ point for $5-25 \%, 2$ points for $25-50 \%, 3$ points for $50-75 \%$, and 4 points for $75-100 \%$. In addition, the scores of the above two items were $0-3$ for low expression and 4-7 for high expression.

\section{Statistical methods}

The data were all processed by the SPSS 16.0 statistical software package, and the measurement data were expressed by $(\bar{x} \pm \mathrm{s})$. The T-test was used to compare the two parameters, and the Spearman test was used to analyze the correlation between IL-17 and VEGF; P $<0.05$ was statistically significant. 


\section{Results}

\section{Identification of $\mathrm{Th} 17$ cells in vitro}

The results of flow cytometry showed that the purity of CD4+ CD62L+ naïve T-cells was more than $90 \%$, and the cells were cultured in vitro for three days with antiCD3, anti-CD28, TGF- $\beta$, and IL- 6 . The size of the CD4+ CD62L+ naïve T-cells was the same under an inverted microscope. After three days of culture, the cells in each group gradually showed colony-like growth and increased cell numbers.

\section{DLBCL mouse model}

The nodule formation rate was $100 \%$ in the mice inoculated with SUDHL-4 cells for approximately eight days. The results showed that eight days was the median tumor onset time T1 of the DLBCL mice, and 28 days was the median tumor survival time T2 of the DLBCL mice.

\section{Comparison and correlation analysis of the expression levels of IL-17 and VEGF in the tumor tissues of the DLBCL mice between the Th17 cell inoculation and control groups}

The IL-17 expression level in T1 and T2 time-points (i.e., the A1 and A2 groups) was higher than that in the control group (i.e., the $\mathrm{B} 1$ and $\mathrm{B} 2$ groups) $(\mathrm{P}<0.05)$. The IL17 expression level in groups A2 and B2 was lower than that in groups $\mathrm{A} 1$ and $\mathrm{B} 1(\mathrm{P}<0.05)$. The results showed that the IL-17 expression level was higher than that of the control group after Th17 cells were inoculated, and the IL-17 expression level was lower with the progress of the tumor course. The VEGF expression level was lower than that of groups $\mathrm{B} 1$ and $\mathrm{B} 2(\mathrm{P}<0.05)$. VEGF expression in groups A2 and B2 was higher than that in groups A1 and $\mathrm{B} 1(\mathrm{P}<0.05)$. It is suggested that the VEGF expression level in Th17 cells is lower than that in the control group and increases with the progression of the tumor (Table 1). The correlation between IL-17 and VEGF expression was analyzed by a Spearman correlation analysis. The difference was statistically significant $(\mathrm{r}=-0.88, \mathrm{P}=0.000)$. It is suggested that there is a significant negative correlation between IL-17 and VEGF (Figure 1).

Expression and correlation of IL-17 and MHC II in the tumor tissue of the DLBCL mice in the Th17 vaccination and control groups

Table 1. Comparison between IL-17 and VEGF in Th17 cell group and control group ( $\mathrm{pg} / \mathrm{ml}, x \pm \mathrm{s})$.

\begin{tabular}{lccccc}
\hline Groups & $\mathrm{n}$ & IL-17 & $\mathrm{P}$ & VEGF & $\mathrm{P}$ \\
\hline Th17 cell group & \multicolumn{4}{c}{0.048} & \multicolumn{2}{c}{0.021} \\
A1 & 10 & $11.84 \pm 0.56^{*}$ & $434.42 \pm 163.25^{\triangle}$ \\
A2 & 10 & $9.56 \pm 0.18^{*}$ & \multicolumn{2}{c}{$594.64 \pm 88.01 \triangle$} \\
Control group & & & 0.032 & \\
B1 & 5 & $9.84 \pm 0.26$ & & $678.16 \pm 141.10$ & \\
B2 & 5 & $7.30 \pm 0.13$ & & $779.91 \pm 166.10$ & \\
\hline
\end{tabular}

*: Comparision between $\mathrm{A} 1$ and $\mathrm{B} 1, \mathrm{P}<0.05$; **: Comparision between $\mathrm{A} 2$ and $\mathrm{B} 2, \mathrm{P}<0.05$. $\triangle$ : Comparision between $\mathrm{A} 1$ and $\mathrm{B} 1, \mathrm{P}<0.05$; $\triangle \triangle$ : Comparision between $\mathrm{A} 2$ and $\mathrm{B} 2, \mathrm{P}<0.05$.

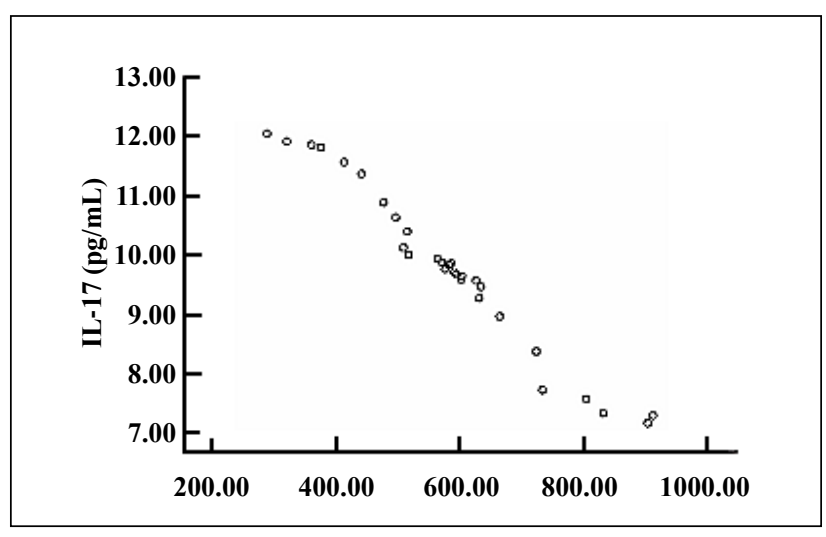

Figure 1. Correlation analysis between IL-17 and VEGF expression in mice.

The IL-17 and MHC II expression levels in the tumor tissues at the T1 and T2 time-points in the Th17 cell vaccination group of DLBCL mice were higher than those of the control group ( $\mathrm{P}<0.05)$, and the IL-17 and MHC II expression levels in the A2 and B2 groups were lower than those in the $\mathrm{A} 1$ and $\mathrm{B} 1$ groups $(\mathrm{P}<0.05)$, which suggests that the IL-17 and MHC II expression levels in the Th17 cell vaccination group were higher than those in the control group, and the IL-17 expression level decreased with the progression of the tumor, as shown in Table 2. The correlation analysis of the IL-17 and MHC II expression levels showed a significant positive correlation between IL-17 and MHC II ( $\mathrm{r}=0.78, \mathrm{P}=0.000)$.

Table 2. IL-17 and MHC II expression in Th17 cell group and control group (pg/ml, $\bar{x} \pm \mathrm{s})$.

\begin{tabular}{llll}
\hline Groups & $\mathrm{n}$ & IL-17(pg/ml) & MHC IIpositive rate (\%) \\
\hline Control group & & & \\
B1 & 5 & $9.84 \pm 0.26$ & 43.59 \\
B2 & 5 & $7.30 \pm 0.13$ & 31.03 \\
Th17 cell group & & & \\
A1 & 10 & $11.84 \pm 0.56^{\star}$ & $68.12^{\triangle}$ \\
A2 & 10 & $9.56 \pm 0.18^{\star *}$ & $53.62 \triangle$ \\
\hline
\end{tabular}

*: Comparision with $\mathrm{B} 1, \mathrm{P}<0.05 ; \triangle$ : Comparision with $\mathrm{B} 2, \mathrm{P}<0.05$.

\section{Discussion}

MIL-17 is mainly secreted by Th17 cells [4]. IL-17 is a kind of proinflammatory cytokine that can cooperate with a variety of cytokines to amplify the inflammatory response. In addition, IL-17 has strong biological functions, such as recruiting neutrophils, promoting the release of inflammatory factors from a variety of cells, promoting cell proliferation, and so forth. Previous studies have shown that IL-17 plays a role in promoting cancer, and the expression of Th17 cells is positively related to tumor microvascular density. IL-17 induces a series of mediators, including VEGF, by acting on stromal cells and fibroblasts, which significantly promotes the development of inflammation and tumor angiogenesis [4]. Although IL-17 is a potential tumor-promoting cytokine, some 
studies have shown that IL-17 can inhibit tumors. It has been found that Th17 polar cells are more effective than Th1 cells in the removal of existing tumors [5]. IL-17 can inhibit the growth of tumors by enhancing CTL activity, such as mast cell tumors, plasmacytomas, and other hematopoietic tumors. Therefore, the relationship between IL17 and tumors is not clear.

VEGF is a kind of multifunctional glycoprotein that is an important factor in the stimulation of the proliferation and migration of vascular endothelial cells. By increasing the mitosis of vascular endothelial cells and promoting the migration of vascular endothelial cells, remodeling the extracellular matrix, and increasing the permeability of blood vessels, VEGF can promote the formation of pathological blood vessels. The formation of tumor blood vessels is conducive to tumor cells infiltrating the lymphatic vessels and promoting the lymph nodes transferring in tumor. Early studies of the analysis of clinical characteristics of non-small-cell lung cancer (NSCLC) patients found that the VEGF expression level will rise with the progress of the clinical stage of NSCLC patients, which suggests that VEGF plays a role in promoting the development of tumors [6]. However, with the development of lung cancer, the increase of VEGF expression in vascular endothelial cells and tumor tissues is related to tumor growth. A study of NHL [3] found that the DFS rate in the group with a high VEGF serum level was significantly lower than that in the group with a low VEGF serum level. A multivariate analysis suggested that the VEGF level was an independent prognostic factor for the total survival time of NHL patients. Kong Jun et al. [7] showed that the positive rate of VEGF in patients with lymphoma was significantly higher than that in healthy controls, which suggests that VEGF can promote the development of lymphoma by promoting the proliferation of vascular endothelial cells. Sun Yan et al. [8] also confirmed that VEGF in NHL can increase vascular permeability, cause changes in extracellular matrix, and thus promote tumor angiogenesis. It can be observed that the increase of the VEGF expression level can promote the growth of NHL.

Previous studies have shown that there is a significant correlation between IL-17, VEGF, and tumor vascular density in NSCLC patients. The IL-17 and VEGF serum levels in NSCLC patients are higher than those in healthy people, which suggests that IL-17 and VEGF may play a regulatory role in the occurrence and development of NSCLC $[9,10]$. Studies in colorectal cancer also showed that IL17 up-regulated VEGF in fibroblasts, thus promoting the formation of fibroblast-induced neovascularization in the process of inflammation and tumorigenesis. Many cancer cells have a high TGF- $\beta$ expression; IL-17 promotes the expression of VEGF, and then in turn promotes the expression of TGF- $\beta$, so it is possible that VEGF mediates angiogenesis [11], and TGF- $\beta$ up-regulates the expression of VEGF receptor in endothelial cells in order to enhance the effect of VEGF on endothelial cells. To summarize, previous studies have found that IL-17 can affect the occurrence and development of tumors by regulating the expression of VEGF in some tumors, but whether the expression of VEGF in DLBCL is regulated by IL-17 to affect the occurrence and development of tumors still lacks relevant research.

Our study found that the IL-17 expression level in the DLBCL mice was higher than that in the control group and decreased with the progression of the tumor; the VEGF expression level in the DLBCL mice was lower than that in the control group and increased with the progression of the tumor. The correlation analysis indicated that there was a significant negative correlation between IL-17 and VEGF, and the high level of IL-17 could downregulate the expression of VEGF. However, with the progression of the disease, the level of IL-17 decreased and the level of VEGF increased, which promoted the growth of the tumor and the death of the DLBCL mice. The negative regulatory mechanism between IL-17 and VEGF has an effect on the occurrence and development of DLBCL tumor.

MHC is a highly polymorphic gene group. The classic MHC class-II gene is composed of three coding genes: HLA-DR, HLA-DQ, and HLA-DP. Its main function is to present antigen peptides to helper T-lymphocytes and to play a role in T-lymphocyte-mediated anti-tumor immunity. Previous studies have shown that the decreased expression of MHC II is related to a variety of tumors, including NHL [12]. During the development of lymphoma, the malignant transformation of lymphocytes into lymphoma cells may be related to the down-regulation of MHC II expression in lymphoma cells [13]. It was discovered in many kinds of lymphoid system tumors, such as borderline lymphoma, which lack MHC II expression [14]; there are also reports that MHC II molecules are related to the pathogenesis of DLBCL, and that MHC II expression may promote tumor infiltration of lymphocytes [15]. MHC class-II molecules may be the immune pathway regulating the development of many tumors, including DLBCL. Studying its possible mechanism can monitor the development of DLBCL and learn the possible immunotherapy pathway.

In this study, the results showed that the IL-17 and MHC II expression levels in the DLBCL mice after Th17 cell inoculation was higher than that in the control group; the IL-17 and MHC II expression levels decreased with the progression of tumor, there was a significant positive correlation between IL-17 and MHC II, and the high level of IL-17 could up-regulate MHC II. However, with the progression of the disease, the level of IL-17 and MHC II decreased, which promoted the growth of the tumor and the death of the DLBCL mice. This is consistent with the previous study that MHC II deficiency promotes tumor development. The Th17 cell's positive regulatory mechanism of the IL-17 and MHC II expression process has an impact on DLBCL tumor development.

However, by what specific pathway does Th17 cells affect the expression of MHC II? Further research is needed to understand whether MHC II can enhance the expression of IL-17 through Th17 cell inoculation and regulate the 
expression level of MHC II in order to prolong the survival period for DLBCL.

\section{Declarations}

Conflict of interest: The authors declare no conflict of interest.

Acknowledgments: This work was supported by Guangdong Medical Science and Research Foundation,China (A2018300); Guangzhou Planned Project of Science and Technology, China(201707010279). The funders had no roles in study design, data collection and analysis, decision to publish, or preparation of the manuscript. The corresponding author had full access to all the data in the study and had final responsibility for the decision to submit for publication.

Authors' contributions: XX and LQS designed and oversaw the project. XX, ZWJ and WT performed experiments and collected data. XX and LQS analyzed and interpreted the data. XX and ZZG provided human tumor cells and tissues. R.A., LY and LF provided technical guidance and critical comments. XX and LQS wrote the manuscript.

\section{References}

1. Tan Xiaoqing,Liu Xiaobo,Gao Xaoyan,et al.Expression and significance of tumor infiltrating lymphocyte in hepatocellular carcinoma.Immunity,2010,11(25):967-972.

2. Zheng Y, Valdez P A, Danilenko D M, et al. Interleukin-22 mediates early host defense against attaching and effacing bacterial pathogens. Nature medicine, 2008, 14(3): 282-289.

3. Niitsu N, Okamoto M, Nakamine H, et al. Simultaneous elevation of the serum concentrations of vascular endothelial growth factor and interleukin- 6 as independent predictors of prognosis in aggressive non-Hodgkin's lymphoma. European journal of haematology, 2002, 68(2): 91-100.

4. Korn T, Bettelli E, Oukka M, et al. IL-17 and Th17 Cells.
Annual review of immunology, 2009, 27: 485-517.

5. Muranski P, Boni A, Antony P A, et al. Tumor-specific Th17-polarized cells eradicate large established melanoma. Blood, The Journal of the American Society of Hematology, 2008, 112(2): 362-373.

6. Wu Xintian,Zhou Shaozhang,Mo Juanmei,et al.Expression and significance of HIF- $1 \alpha, \mathrm{VEGF}$ and TGF- $\beta$ in NLSLC. China Lung Cancer,2013,15(1):46-49.

7. Kong Jun,Zhou Min.Expression and significance of VEGF and p53 in serum of NHL patients.Modern Oncology,2011,19(12):2543-2545.

8. Sun Yan,Chu Xiaoxia,Han Xiuhua, et al.Expression and significance of VEGF and COX-2 in NHL. J Chin Physician,2010,12:1623-1627.

9. Kirshberg S, Izhar U, Amir G, et al. Involvement of CCR6/ CCL20/IL-17 axis in NSCLC disease progression. PLoS One, 2011, 6(9).

10. Chen X, Wan J, Liu J, et al. Increased IL-17-producing cells correlate with poor survival and lymphangiogenesis in NSCLC patients. Lung cancer, 2010, 69(3): 348-354.

11. Jeon S H, Chae B C, Kim H A, et al. Mechanisms underlying TGF- $\beta 1$-induced expression of VEGF and Flk-1 in mouse macrophages and their implications for angiogenesis. Journal of leukocyte biology, 2007, 81(2): 557-566.

12. Chamuleau M E D, Ossenkoppele G J, Van De Loosdrecht A A. MHC class II molecules in tumour immunology: prognostic marker and target for immune modulation. Immunobiology, 2006, 211(6-8): 619-625.

13. Plander M, Seegers S, Ugocsai P, et al. Different proliferative and survival capacity of CLL-cells in a newly established in vitro model for pseudofollicles. Leukemia, 2009, 23(11): 2118-2128.

14. Bernd H W , Ziepert M, Thorns C , et al. Loss of HLA-DR expression and immunoblastic morphology predict adverse outcome in diffuse large B-cell lymphoma - analyses of cases from two prospective randomized clinical trials. Haematologica, 2009, 94(11):1569-1580.

15. Bushway M, Cycon K A, Mulvaney K, et al. Coordinate loss of MHC class II expression in the diffuse large B cell lymphoma cell line OCI-Ly2 is due to a novel mutation in RFX-AP. Immunogenetics, 2010, 62(2): 109-116.

Cite this article as: $\mathrm{Xu} \mathrm{X}$, Wei $\mathrm{T}$, Zhong $\mathrm{W}$, et al. IL-17 regulates the expression of major histocompatibility complex II and VEGF in DLBCL mice on tumor growth[J]. Aging Pathobiology and Therapeutics, 2020, 2(2): 96-100. 